JAVIER CORREDOR*

JORGE MAHECHA"

ANA LuCía TRUJILLO

\title{
REFORMA ESCOLAR Y FORMACION DOCENTE EN E-LEARNING: EL CHOQUE DE LAS TRADICIONES
}

\begin{abstract}
Resumen
Este artículo revisa el papel que el choque de tradiciones y la competencia entre diversas fuentes de legitimidad puede tener en el éxito o fracaso de los procesos de formación docente y reforma escolar en e-learning. Mediante un estudio cualitativo, este artículo muestra cómo prácticas que son legítimas para el Ministerio de Educación no coinciden necesariamente con prácticas que tienen alta legitimidad a nivel regional. En particular, se revisa la manera en que programas de formación docente en e-learning, diseñados en colaboración con el Ministerio de Educación Nacional, pierden rápidamente legitimidad frente a los actores encargados de financiar proyectos en los departamentos. Adicionalmente, el estudio muestra que existe una tensión entre una lógica pedagógica y una lógica tecnológica en los procesos de formación docente y reforma escolar en e-learning. Esta tensión hace que los resultados de la investigación básica en cognición tengan dificultades para impactar las prácticas de formación en las regiones.
\end{abstract}

Palabras clave: Reforma escolar, formación docente, e-learning, TIC, cognición distribuida.

\section{REFORMA ESCOLAR Y FORMAÇÃO DOCENTE EM E-LEARNING: O CHOQUE DAS TRADIÇÕES}

\section{Resumo}

Este estudo explora as fontes de legitimidade sob os quais os programas de formação de professores do e-learning têm que atuar. Tendo por base dados qualitativos procedentes de quatro regiões da Colômbia, a análise dos resultados sugerem que as instituições regionais responsáveis dos programas de formação de professores respondem ás formas de legitimidade aceitas em suas regiões. Contudo, ditas formas de legitimidade competem com os objetivos da reforma, da maneira em que foram planejados no nível nacional. A legitimidade no nível regional (estado/província/departamento) provém das tradições e das perspectivas populares da pedagogia e do e-learning, as quais não coincidem com as perspectivas promovidas pelos responsáveis políticos no nível nacional. Esta competição entre fontes de legitimidade regionais e nacionais faz com que a reforma educacional seja ineficaz. Para exemplificar este conflito, este estudo revê e compara duas tradições a respeito do e-learning: a primeira, concentra-se nos aspectos tecnológicos dos computadores, e a segunda, foca-se nos aspectos pedagógicos relacionados aos Ambientes Virtuais de Aprendizagem (AVAs).

* Profesor asistente del Departamento de Psicología de la Universidad Nacional de Colombia. PhD en Estudios Cognitivos en Educación de la Universidad de Pittsburgh.

Correo electrónico: jacorredora@unal.edu.co

** Investigador y coordinador del proyecto en el Centro de Investigación y Formación en Educación, Cife, de la Universidad de los Andes. Magíster en Educación de la Universidad de los Andes.

Correo electrónico: jorgeal.mahecha@gmail.com

*** Investigadora en el Centro de Investigación y Formación en Educación, Centro de Investigación y formación en educación (CIFE), de la Universidad de los Andes. Magíster en Educación de la Universidad de los Andes.

Correo electrónico: al.trujillo97@uniandes.edu.co

Artículo recibido el 15 de abril de 2009 y aprobado el 15 de mayo de 2009

Pedagogía y Saberes N. ${ }^{28}$. Universidad Pedagógica Nacional. Facultad de Educación, 2008, pp. 121-130 
Palavras-Chaves: Ambientes virtuais de aprendizagem (AVAs), E-learning; Formação de professores, Cognição distribuída.

\title{
SCHOOL REFORM AND TEACHER EDUCATION IN E-LEARNING: THE CLASH OF TRADITIONS
}

\begin{abstract}
Using qualitative data from four regions of Colombia, this study explores the sources of legitimacy which teacher education programs in e-learning have to respond. Results suggest that regional institutions in charge of teacher education programs respond to forms of legitimacy that are accepted in their regions, but not necessarily coincides with practices which have high legitimacy at national level. Legitimacy at the regional level (State/Province/Department) comes from traditions and folk perspectives of pedagogy and e-learning that do not coincide with the perspectives held by policy-makers at the national one. This competition between regional and national sources of legitimacy causes that reforms at the school are not effective. To exemplify this clash of legitimacy sources, this study reviews and compares two traditions regarding e-learning: one that focuses on the technological aspects of computers and other one that focuses on the pedagogical aspects of virtual learning environments.
\end{abstract}

Key Words: School reform, teachers education, E-Learning, virtual learning environments, Distributed cognition.

\section{INTRODUCCIÓN}

Cuando Larry Cuban (1993) publicó su artículo "Los computadores se enfrentan al aula: el aula gana1", pocos podían predecir que éste se convertiría en un clásico. Sin embargo, hoy nadie duda de la vigencia de las preguntas allí expresadas. Este texto mostraba que los ambientes educativos estaban rezagados en relación con otros espacios sociales en su capacidad para integrar los computadores en sus prácticas cotidianas y cuestionaba la esperanza fácil en que la tecnología liberaría a la educación de sus males más enraizados. En un intento por explicar el bajo impacto aún existente de los computadores en la enseñanza, este artículo revisa un programa de impacto nacional, planeado y coordinado por el Ministerio de Educación, que buscaba crear cinco centros de formación docente y reflexión pedagógica en cuatro regiones del país ${ }^{2}$. A partir

1 El título original del articulo era "Computers meet classroom: classroom wins". Lo que este título sugiere es que, a pesar del encuentro entre computadores y aula, las aulas imponen sus prácticas en vez de modificarlas.

2 Este estudio fue realizado gracias a la financiación otorgada por la Fundación Empresarios por la Educación y el Ministerio de Educación a Jorge Mahecha como coordinador del proyecto en el de estas experiencias, este artículo revisa el posible papel de las tradiciones regionales en el éxito o fracaso de los computadores como herramientas de transformación pedagógica.

\section{RELEVANCIA Y RETOS DEL ESTUDIO DE LA REFORMA EN E-LEARNING}

Las transformaciones tecnológicas ocurridas en los últimos veinte años han ampliado las posibilidades de acceso y difusión de información a nivel global, al mismo tiempo que han creado urgentes necesidades sociales a nivel local. Para responder a éstas, los encargados de la política pública han respondido con agresivos programas sociales y educativos que buscan proveer la infraestructura necesaria y capacitar a los ciudadanos con habilidades informáticas básicas. Particularmente importantes en este espectro de programas son los programas de reforma escolar y formación docente en e-learning, ya que éstos median entre la adquisición de infraestructura y dotación básica y los

Centro de Investigación y Formación en Educación, (CIFE), de la Universidad de los Andes. efectos reales sobre las competencias tecnológicas de los estudiantes. Dada la naturaleza experimental y la presión política por resultados positivos, ha sido siempre difícil establecer protocolos de evaluación claros para este tipo de programas, o más aún identificar los determinantes de éxito para la formación docente en e-learning.

Este artículo describe la evaluación de un proyecto de formación de docentes en e-learning y nuevas tecnologías para el aprendizaje. Mediante un análisis cualitativo, este estudio propone que los programas de formación docente en elearning ejemplifican un caso de choque de tradiciones a un nivel pedagógico y político. Los autores se fundamentan en la teoría institucional para entender los diferentes niveles y perspectivas que interactúan en los procesos de reforma escolar. Para ejemplificar cómo el choque de tradiciones a un nivel pedagógico obstaculiza la aprehensión de resultados provenientes de la investigación básica, este artículo describe la dificultad que la cognición distribuida ${ }^{3}$ y sus conse-

3 Cognición distribuida se refiere aquí a las perspectivas que en la cognición contemporánea propenden a la inclusión de los recursos físicos y socioculturales 
cuencias para el e-learning en cuanto al impacto en las prácticas y el discurso docente.

\section{LAS LIMITACIONES DE LA REFORMA ESCOLAR}

La reforma escolar, entendida en un sentido amplio, incluye todas las acciones diseñadas y propuestas por los niveles administrativos y políticos del sistema educativo dirigidos a modificar positivamente las condiciones y resultados de los procesos educativos. En este sentido, la reforma escolar incluye acciones a diferentes niveles, desde la publicación de estándares y mapas de ruta para el sistema educativo hasta los procesos de formación docente y evaluación escolar. Dicho de otro modo, la reforma escolar se refiere al conjunto de acciones que buscan traducir la visión contenida en la política pública en cambios efectivos en las prácticas de aula.

Una visión cándida de la reforma escolar supone que las políticas educativas impactan las prácticas de aula tal como han sido pensadas en los altos círculos de decisión. Más aún, esta visión supone que las propuestas educativas llegan a las aulas tal y como han sido desarrolladas en los centros de investigación. En esta visión, la ruta de desarrollo de la reforma escolar va de los centros de investigación a los centros de decisión y de allí a las prácticas de aula. Sin embargo, esta visión olvida que en el proceso de transformación educativa participan múltiples procesos mediacionales e interactúan diferentes actores que, en muchos casos, difieren en sus perspectivas frente a la realidad educativa (Light, Money, Heinze, Brunner, Wexlar, Mandinach y Fasca, 2005). Para responder a esta dificultad, ha sido frecuente intentar elaborar currículos a prueba de maestros (Bautista, 1999 y

en la descripción de los procesos de razonamiento. No debe confundirse con procesamiento distribuido, que es una de las denominaciones que se ha dado al modo de funcionamiento de las arquitecturas cognitivas que usan modelos conexionistas.
Díaz, 1997). Esta visión, centrada en la reforma "desde arriba", ha mostrado ser ineficaz para impulsar procesos de cambio pedagógico, ya que normalmente las comunidades educativas reaccionan de maneras que desafían las predicciones hechas por los reformadores (Berman y Mclauglin, 1976). Por un lado, en cualquier ambiente educativo hay prácticas y creencias preexistentes, diversas culturas escolares, que no necesariamente coinciden con la visión y los objetivos de la reforma y los reformadores (Hubbard, Stein y Mehan, 2006); por el otro, las comunidades educativas resisten activamente la interferencia e imposición vertical de contenidos y metodologías (Torres, 2000).

La realidad es que en el proceso de mediación los resultados de investigación son redefinidos por las personas encargadas de delimitar la política pública, por los actores encargados de llevar dichos contenidos a los docentes y por los maestros al momento de implementar prácticas de aula. Este proceso interactivo entre tradición e innovación, entre las prácticas existentes y los objetivos de la reforma es el que en últimas define los resultados de los procesos de reforma (Halverson, Kelley y Kimball, 2004).

\section{TEORÍA INSTITUCIONAL Y REFORMA ESCOLAR}

Una herramienta teórica que puede ser útil para entender los efectos reales de los intentos de reforma escolar y formación docente sobre las prácticas de aula es la teoría institucional (Meyer y Rowan, 1977). La teoría institucional plantea que las respuestas que las organizaciones, incluyendo los centro educativos, dan a los intentos de reforma y a las presiones provenientes de los sistemas políticos y administrativos deben ser entendidas atendiendo a la interacción entre dos niveles: el ambiente institucional y el ambiente técnico. El ambiente institucional se refiere al nivel formal de definición de la organización que se asienta en los niveles políticos y administrativos y en las relaciones con otras organizaciones o instituciones con las que interactúa la institución educativa. En la definición formal de la estructura de la escuela se encuentran los productos institucionalizados, los servicios y los propósitos políticos y educativos tal y como han sido definidos en la normatividad. La normatividad, sin embargo, se transmite mediante sistemas simbólicos que se instalan espontáneamente en el contexto social e institucional y que constituyen fuentes de legitimidad para las organizaciones. Estos sistemas simbólicos se convierten en tradiciones y rituales y son los que en últimas terminan validando la existencia y continuidad de las prácticas establecidas en las organizaciones (Maguire y Uchida, 2000). El ambiente técnico, por otro lado, se refiere a la práctica pedagógica que se produce en el aula; es denominado técnico no porque presuponga una práctica mecánica de parte del docente, sino porque implica un saber hacer relacionado directamente con acciones en un nivel concreto. El ambiente técnico es el día a día de la práctica educativa, es el proceso de enseñanza y evaluación y las acciones concretas de los docentes frente a los dilemas que enfrentan cotidianamente.

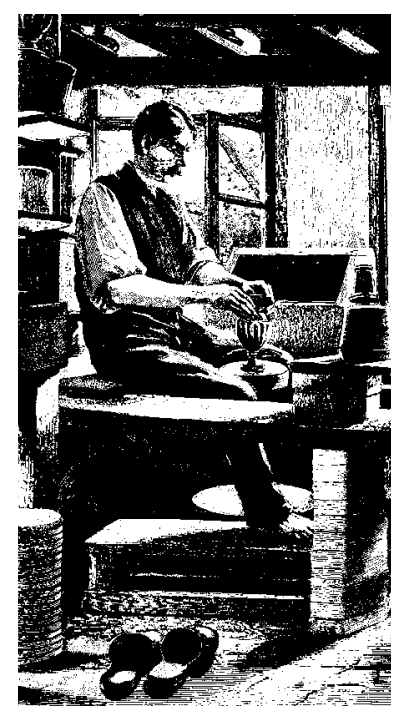

El conflicto que la teoría institucional presenta es que la legitimidad de la práctica pedagógica viene de rituales ("ambiente institucional") que no tienen que ver con la enseñanza ("ambiente técnico”) (Rowan y Miskel, 1999). La norma convertida en ritual es inefectiva para modificar las prácticas pedagógicas, ya que sus alcances se reducen al 
espacio del "mito y la ceremonia" (Meyer y Rowan, 1977). Esto permite, por ejemplo, que una institución educativa pueda ser altamente exitosa en un proceso de reforma sin tener que modificar su actividad en el aula, respondiendo a la reforma con la adopción pública de ciertos rituales y postulados formales. Un concepto básico de la teoría institucional es la disociación (decoupling) (Coburn, 2004 y Firestone, 1985). La disociación se produce cuando las escuelas separan lo que sucede en el nivel institucional de lo que sucede en el nivel técnico debido a la presión por cumplir objetivos múltiples y en muchos casos contradictorios. En otras palabras, ante la presión por producir resultados y legitimarse públicamente, los colegios separan su práctica pedagógica de los rituales con los que se legitiman en su espacio social e institucional. Según Coburn (2004), la teoría institucional, en su versión más clásica, sostiene que la disociación se produce para proteger el núcleo técnico de escrutinio externo o para responder a presiones y objetivos diversos, pero coalescentes. La disociación sucede, por ejemplo, cuando los colegios responden a la creación de estándares de evaluación o de exámenes de control con prácticas que se enfocan exclusivamente en un entrenamiento para el test, pero que no modifican los procesos instruccionales en el aula. En otro orden del mismo fenómeno, la disociación se puede ver en la reciente tendencia a remplazar modificaciones reales en las prácticas pedagógicas con la adopción de protocolos de calidad tomados de los procesos industriales; dichos protocolos de calidad permiten responder al control estatal y a los procesos de acreditación a un nivel simbólico, pero no necesariamente modifican las prácticas de enseñanza. Aunque el esfuerzo que se encuentra detrás de los intentos de asumir protocolos de calidad es loable, su efecto sobre las prácticas de aula es incierto; esfuerzos análogos en el pasado resultaron inefectivos. Son en particular mal recordados, para citar sólo un ejemplo, la codificación de objetivos y el uso de parceladores implementados en el paradigma de la tecnología educativa.
¿Cómo encaja la teoría institucional y la idea de la disociación en el análisis de la reforma en e-learning? En este caso, la propuesta concreta es que los mediadores de la reforma, las universidades encargadas de llevar a cabo los cursos de formación docente, deben responder, por un lado, a las presiones de los centros administrativos y de decisión política a nivel nacional, pero también deben responder a los sistemas de legitimidad existentes en sus regiones. En este doble juego, los procesos de reforma adscriben su discurso y sus prácticas al sistema de legitimidad que les produce réditos directos: el sistema de legitimidad local. Como se verá más adelante, la consistencia entre el discurso sostenido por la institución encargada de llevar a cabo el proceso de formación docente, y el discurso dominante a nivel local $^{4}$ es clave para la supervivencia de los procesos de reforma. Cuando la reforma es consistente con el discurso central-nacional, pero inconsistente con el discurso local, el proyecto desaparece. Esto sucede porque es el ambiente institucional local el que provee los recursos a la institución encargada de llevar a cabo la formación docente. En este sentido, la propuesta de este estudio se inscribe en la tendencia reciente a revisar la teoría institucional (Coburn, 2004). En particular, se intenta aquí revisar la concepción de ambiente institucional y de las fuentes de legitimidad allí implicadas. En la sección de resultados se sugerirá que el ambiente institucional no puede ser considerado como homogéneo y que, por el contrario, los actores educativos están sujetos a presiones que vienen de diversas organizaciones sociales.

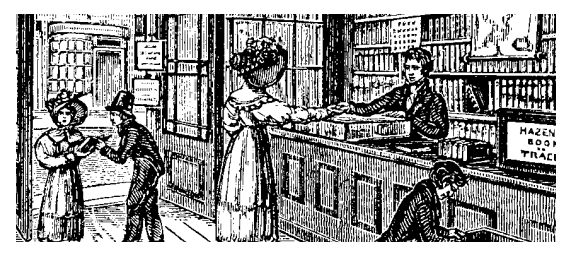

4 Discurso local se refiere al discurso dominante a nivel departamental y municipal. Discurso nacional se refiere a la propuesta pedagógica en e-learning como ha sido pensada en entes de carácter nacional (e. g., Ministerios).

\section{¿PARA QUÉ REFORMAR? INVESTIGACIÓN BÁSICA Y REFORMA ESCOLAR}

Hasta aquí, se ha presentado una visión de los efectos de la reforma escolar desde la teoría institucional. Esta visión, en alguna medida, se construye sobre el supuesto implícito de que la reforma educativa va a producir efectos positivos en los procesos de enseñanza y en la formación de los estudiantes resultante de ésta. Esta idea, sin embargo, puede ser reformulada en términos más específicos: se sostiene aquí que la reforma escolar es positiva para los procesos educativos porque puede, en cierta manera, acercar los resultados de la investigación básica en psicología cognitiva a los procesos de aula. En años recientes, la psicología cognitiva ha producido resultados sorprendentes para la comprensión de los procesos de aprendizaje individual y colectivo. Entre estos descubrimientos se encuentran las consecuencias de la integración proposicional a nivel de lectura y aprendizaje (Johnson-Laird, 1980 y Kintsch, 1988); la función de los esquemas previos en el desarrollo y la construcción de nuevos aprendizajes y, derivado de esto, la importancia de la representación de la tarea y la identificación de esquemas adecuados en la resolución de problemas (Simon y Hayes, 1976 y Perkins y Salomón, 1992) y la centralidad de los procesos colaborativos en el desarrollo de las capacidades de razonamiento y lectura (Palinscar y Brown, 1984). Estos descubrimientos pueden, dadas las condiciones adecuadas de implementación, modificar el panorama y los procesos educativos en el aula. Para citar sólo un ejemplo, la idea de que la integración proposicional se produce únicamente cuando los estudiantes cambian una representación literal de las premisas por una representación integradora de éstas, va en contravía de prácticas todavía extendidas, como la repetición literal de textos para generar memoria exacta de contenidos. En este sentido, una reforma educativa fundamentada en datos y en los resultados de la investigación básica sería benéfica para la educación en su conjunto. 
Sin embargo, la relación entre investigación básica y prácticas de aula ha sido objeto de debate. No es que en general se considere que los resultados de este tipo de transferencia de conocimiento sean negativos para los procesos educativos, sino que se duda del impacto real que estas teorías, construidas en el laboratorio u otros ambientes artificiales, puedan tener en el aula. En el lado optimista de este debate se encuentran quienes consideran que los principios derivados de la teoría cognitiva pueden ser aplicados directamente al diseño de ambientes educativos (Lovett y Greenhouse, 2000). En el lado escéptico, están quienes se enfocan en las restricciones que previenen una adecuada transferencia de conocimiento desde la investigación básica a los ambientes aplicados. Estos estudios revisan los factores que generan ruido en la lectura que los actores en el campo hacen de los resultados producidos en la academia; un ejemplo de esta perspectiva son los estudios que muestran las concepciones que diversos actores en el campo educativo tienen de lo que es usar adecuadamente la evidencia científica (Coburn y Talbert, 2006). Existe finalmente un línea moderada, por decirlo de algún modo, que considera que una adecuada transferencia de los descubrimientos producidos en ciencia básica a los ambientes aplicados pasa por un proceso interactivo de investigación en el laboratorio, aplicación en el aula y rediseño a partir de la experiencia en el campo (Klahr y Li, 2005). Para entender mejor las implicaciones de estas tres perspectivas, es necesario mostrar las consecuencias que la investigación básica puede tener para el e-learning ${ }^{5}$. En la siguiente sección, se señalan las consecuencias que el desarrollo de

5 En este texto, el término e-learning se usa en un sentido amplio, que incluye los diferentes usos que se les dan a las Tecnologías de Información y Comunicación en contextos educativos. En este sentido, e-learning se refiere aquí a prácticas diversas, como la educación a distancia, la enseñanza híbrida respaldada por computador (blended learning), y la participación en prácticas colectivas de construcción de conocimiento, como los foros virtuales. la cognición distribuida tiene para la enseñanza respaldada por las nuevas Tecnologías de la Información y la Comunicación.

\section{COGNICIÓN DISTRIBUIDA EN E-LEARNING}

Uno de los desarrollos con un impacto potencial más alto para el uso de herramientas tecnológicas en el aula es el desarrollo del la cognición distribuida como un concepto fundamental en la explicación del razonamiento en situaciones naturales. Esta noción es entendida aquí en un sentido amplio que abarca dos perspectivas teóricas cuyas diferencias van más allá del alcance de este texto, pero que están unificadas por la idea de que el entendimiento de los procesos cognitivos tiene que ir más allá del individuo para incluir recursos ambientales y significados culturales. La primera perspectiva propone que la unidad de análisis del proceso cognitivo debe ampliarse para incluir a los equipos de trabajo y sus ambientes de tarea (Hutchins, 1995); la segunda perspectiva, que se ha denominado perspectiva situada, asume que los procesos cognitivos necesitan ser entendidos en función de los significados sociales y culturales que rodean las acciones de las personas y de las comunidades de práctica en las cuales esas acciones se producen (Greeno, Collins y Resnick, 1996).

Para el e-learning, los desarrollos de la cognición distribuida implican que los estudiantes deben ser educados y evaluados en ambientes que incluyan y utilicen las características de los ambientes culturales. Esto por ejemplo implica el uso transversal de evaluaciones con libro abierto y acceso a Internet ${ }^{6}$ (Herington \& Herrington, 1998). Implica también permitir que los estudiantes usen recursos informáticos para el cálculo y la realización de tareas procedurales como calculadoras, programas estadísticos y sistemas diseñados para resolver automáticamente ecuaciones y problemas de algebra (Drijvers y Van

6 OBOW, Open Book, Open Web, por sus siglas en inglés (Willliams, 2006).
Herwaarden, 2001, Guin y Trouche, 1999 y Heid, 1988) Adicionalmente, sugiere que tanto profesores como estudiantes construyan comunidades de aprendizaje fundamentados en los recursos informáticos disponibles; por ejemplo, usando tableros de discusión, wikies o blogs compartidos para discutir problemas educativos y teóricos (Matusov, Hayes y Pluta, 2005 y Schuck, 2003). La cognición distribuida enseña que el aprendizaje y la resolución de problemas se ven favorecidos cuando se distribuye la actividad cognitiva entre el sujeto y los recursos materiales y socioculturales que lo rodean y, sobre todo, que sintonizando las prácticas educativas con los recursos - humanos y materiales-disponibles en los contextos socioculturales, los estudiantes adquieren herramientas de alta transferencia a ambientes reales (Greeno et al., 1996). Por ejemplo, memorizar información o realizar cálculos estadísticos a mano no tiene sentido cuando el ambiente real en el que se van a utilizar estas habilidades provee sistemas de búsqueda de información vía Internet y paquetes estadísticos que pueden realizar en segundos los procedimientos que a los estudiantes les llevarían días.

Hasta aquí, se ha planteado que la teoría institucional puede ser una herramienta para entender los efectos de los procesos de formación docente en e-learning. Se plantea, sin embargo, que, a diferencia de como se hace en las versiones tradicionales de la teoría institucional, el ambiente institucional que rodea el proceso de reforma en e-learning en Colombia no puede ser caracterizado como homogéneo. Dada la tensión entre una tradición de control nacional y los procesos de descentralización llevados a cabo en los últimos veinte años, los actores encargados de implementar la reforma deben responder a las presiones de dos ambientes institucionales cuyas perspectivas no necesariamente coinciden: el ambiente institucional nacional y el ambiente institucional regional. En resumen, se ha sugerido que los procesos de reforma escolar pueden ser benéficos para los actores educativos porque acercan los resultados de investigación básica a los ambiente escolares. Se ha utilizado la 


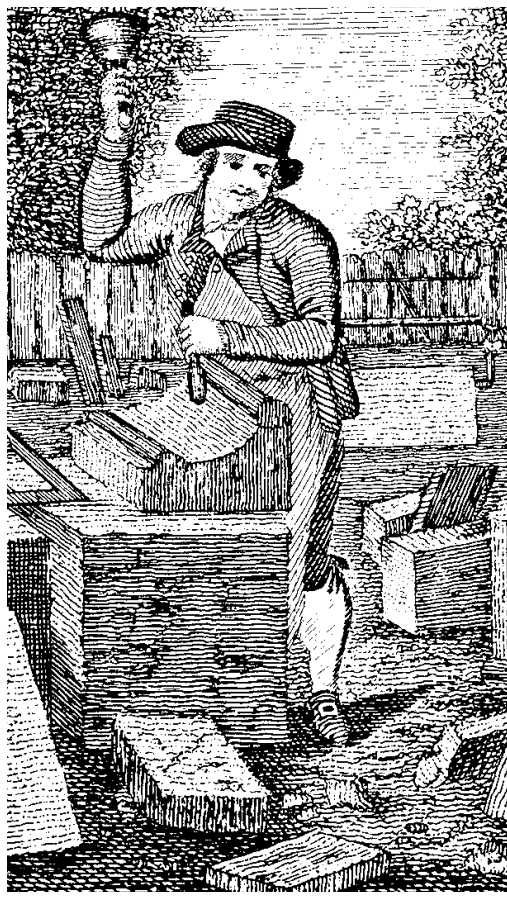

cognición distribuida como un ejemplo de los efectos que pueden tener los resultados de la investigación básica en los procesos educativos respaldados por computador. Ahora, se describe el método de estudio que permitirá ver cómo los conceptos antes expuestos se presentan en cinco situaciones de reforma escolar en e-learning en el contexto colombiano.

\section{CARACTERÍSTICAS DEL PROGRAMA DE FORMACIÓN DOCENTE}

La metodología utilizada en este estudio está intrínsecamente relacionada con las características del programa de formación docente al que se dirigía la investigación. Por esto, antes de pasar a la descripción de los niveles en los cuales se recolectaron los datos, es necesario describir las características del programa Academias $\mathrm{TI}$, al cual se dirigía la evaluación presentada en este artículo. El programa Academias TI tenía como objetivo crear centros a nivel regional que, por un lado, cumplieran funciones de formación docente en integración de TIC en sus prácticas de aula y, por el otro, sirvieran como espacios de reflexión e investigación sobre el quehacer pedagógico apoyado por las nuevas tecnologías. El programa fue avalado por el Ministerio de Educación en convenio con Microsoft y la Fundación Empresarios por la Educación. La idea era proporcionar apoyo económico y logístico a universidades regionales para que ellas crearan dichos centros. El programa proporcionaba una financiación para la primera cohorte de docentes formados y aspiraba a que los centros buscaran financiación por medio de las secretarías departamentales y municipales de educación para mantener su continuidad. En la primera fase del proyecto, se realizó una convocatoria nacional a partir de la cual fueron seleccionadas las universidades que llevarían a cabo la formación docente en las regiones. Dichas universidades participaron en diversas actividades en las que recibieron apoyo y capacitación tanto a nivel pedagógico como tecnológico. A partir de ese punto, las universidades realizaron el proceso de implementación del programa a nivel regional. Este proceso consistió inicialmente en la capacitación de una cohorte de docentes de primaria, media y básica que constituyeron la población beneficiaria del programa. A partir de allí, las universidades debían buscar financiación para darle continuidad al centro y a los programas de formación docente.

\section{MÉTODO}

Este estudio utilizó una metodología cualitativa que organizó la información proveniente de los documentos base en cinco estudios de caso, uno para cada uno de los proyectos evaluados a nivel regional. Estos estudios de caso presentaban información sobre los aspectos pedagógicos de los procesos de formación docente en cada centro y sobre los aspectos administrativos implicados en la coordinación y gestión de los proyectos. Dichos estudios fueron contrastados en diversas dimensiones tentativas hasta refinar la interpretación de los datos en las dimensiones de análisis que se presentan en la sección de resultados. Se puede afirmar, entonces, que este es un estudio de naturaleza comparativa, basado en metodologías de recolección y procesamiento de información de tipo cualitativo. En lo que respecta al carácter comparativo de este estudio, se entiende así en virtud a una definición de este campo de estudio que incluye investigaciones que organizan casos diversos en torno a dimensiones comparables para producir conclusiones que se extiendan más allá del caso particular (Phillips y Schweisfurth, 2006). En particular, aunque este no es un estudio internacional, sí pretende mediante la comparación establecer conclusiones que, aunque no necesariamente extrapolables a otros contextos, presenten utilidad para interpretar situaciones similares en las que existan procesos de formación docente en el uso de TIC. Con respecto a los casos particulares analizados aquí, cada uno de ellos fue examinado usando diversas herramientas de recolección de información cualitativa dirigidas a describir diversos niveles de actuación del programa Academias $\mathrm{TI}$, con el fin de contrastar el programa pensado con el programa efectivamente ejecutado (Espejo, Schuhmann, Schwaninger y Bilello, 1996).

Para el análisis del programa pensado se acudió al análisis de diversas fuentes de información que describían el programa, su gestión e implementación a nivel nacional y regional. Todas las fuentes de información fueron obtenidas en visitas de campo a las cuatro regiones implicadas y a las instituciones encargadas de financiar los programas a nivel nacional en Bogotá. Estos documentos incluían los convenios marco de actuación entre las universidades y el Ministerio de Educación y, para cada universidad, los planes de estudio propuestos en cada uno de los programas. En lo que respecta al análisis del programa ejecutado, se realizaron entrevistas en profundidad y grupos focales con diversos actores del proceso.

La integración de estas perspectivas permitió construir un panorama integrado de los diversos enfoques involucrados en el desarrollo del programa, los cuales configuraron los niveles de análisis nacional y regional de comparación, de manera análoga a como se establecen categorías emergentes en el 
análisis cualitativo, en particular, en lo que se conoce como grounded theory (teoría fundamentada) (Strauss y Corbin, 1998). A nivel nacional, los documentos recolectados fueron los términos de referencia de la convocatoria para el programa, las evaluaciones realizadas a los actores regionales, y los diseños curriculares utilizados para formar a los actores regionales (e. g., las universidades) encargados de llevar a cabo el proceso de reforma. Asímismo, se realizaron reuniones con los encargados del programa en el Ministerio de Educación y en la entidad financiadora.

A nivel regional, los datos se organizaron en tres niveles: decisión regional, programas de formación docente y beneficiarios. Los documentos recolectados en el nivel de decisión regional fueron los convenios entre las universidades encargadas de llevar a cabo el programa y las secretarías de educación de los departamentos y municipios involucrados. A este nivel, se entrevistaron los encargados de las áreas de nuevas tecnología y e-learning en las secretarías de educación regionales. Para los programas de formación docente, se recolectaron los planes curriculares de los cursos de formación docente y ejemplos de las actividades (e. g., talleres) utilizados en las sesiones de trabajo con los profesores), se realizaron grupos focales y entrevistas semiestructuradas con los coordinadores y los instructoresprofesores universitarios encargados de la formación de los docentes de básica y media; adicionalmente, se observaron sesiones de formación docente cuando este proceso aún se encontraba en curso. A nivel de los beneficiarios, los documentos recolectados fueron los productos de los talleres y las actividades diseñadas por los docentes; se realizaron grupos focales con los docentes beneficiarios y se realizaron observaciones de clase.

\section{RESULTADOS}

Los resultados que aquí se presentan se organizan en torno a tres ejes analíticos construidos a partir de la información anteriormente descrita. El primero de ellos se denomina Legitimidad regional versus legitimidad nacional. Aquí se aborda la manera en que las fuentes de legitimidad regionales son centrales para las instituciones encargadas de la formación docente. El segundo eje, denominado Lógica tecnológica versus lógica pedagógica, expone cómo en los procesos de reforma en las regiones existe una tensión latente entre una visión de la reforma y, por tanto, de la formación docente, centrada en lo puramente tecnológico y una visión que se concentra en la modificación de las prácticas pedagógicas a partir de la inclusión de la tecnología en el aprendizaje formal e informal. El tercer eje se denominó El espacio físico de la reforma y los límites de la cognición distribuida. En esta categoría se describe el modo en que, debido a la interacción entre el poder de las instituciones financiadoras regionales y la prevalencia de una visión de la reforma centrada en lo tecnológico, las propuestas de la cognición distribuida no parecen tener impacto en la mayoría de las regiones incluidas en este estudio. Finalmente, se realiza una revisión de las implicaciones que estos resultados tienen para los procesos de reforma escolar y formación docente en e-learning y para los procesos de cambio pedagógico en el contexto de las nuevas tecnologías.

\section{Repensando el ambiente institucional: Legitimidad regional versus legitimidad nacional}

"Este año hubo una convocatoria que comenzó en marzo, inscribimos el proyecto porque tiene que pasar por convocatoria para que tenga la disponibilidad presupuestal, sin embargo los proyectos para la secretaría no pasaron. Me parece que hubo un desconocimiento por parte de la secretaria actual de ese proyecto en relación con el Ministerio" (Región 1: entrevista con actor del nivel formación docente).

"El inconveniente con la secretaría es que a veces no hay coherencia entre las políticas y la difusión de esas políticas. Por un lado, el Ministerio está haciendo un trabajo de implementación de nuevas tecnologías, un trabajo en los departamentos, pero de pronto las secretarías no tienen la información completa" (Región 2: entrevista con actor del nivel formación docente).
En las versiones clásicas de la teoría institucional, el ambiente institucional se ha considerado como una fuente más o menos homogénea de rituales y formas de legitimación que emana de la definición formal de la organización. Sin embargo, los resultados de este estudio muestran que la legitimidad de los programas de formación docente emana de dos fuentes diferentes. Por un lado, los programas de formación docente deben responder ante los actores nacionales que definen la política pública y diseñan iniciativas de impacto nacional como la estudiada en este proyecto. Por el otro, los programas de formación docente también deben construir su legitimidad frente al nivel de decisión regional, en este caso, las secretarías de educación municipales y departamentales que proveen los recursos económicos para la continuidad de los programas. Esta doble naturaleza hace que los programas de formación docente deban decidir a cual lógica deben adscribirse. En los cinco casos estudiados, los actores de los programas de formación docente reportaron instancias de negociación con los actores encargados de la decisión a nivel regional, como se ve en los fragmentos señalados al comienzo de esta sección. En los casos en que se reportó conflicto en esta negociación, los programas de formación docente se vieron interrumpidos.

\section{Formación docente: Lógica tecnológica versus lógica pedagógica}

\begin{abstract}
"Yo creo que lo más difícil, el caballo batalla, ha sido siempre que ellos entiendan de que este no es un curso de herramientas, sino una propuesta clara de crear ambientes de aprendizaje mediado por el uso de la tecnología. Ellos vienen acá con la idea muy clara de decir que se van a volver expertos en Cmap, en wiki y pues ya uno pues con el trabajo pedagógico que hace uno logra desmontarles ese mito [...]" (Región 3: grupo focal con actor del nivel formación docente).
\end{abstract}

El segundo resultado claro en esta investigación es el conflicto existente entre dos lógicas relativas al proceso de formación docente en e-learning: una que 
privilegia el desarrollo de competencias tecnológicas y otra que se concentra en los aspectos pedagógicos relativos al proceso de reforma. Esta tensión es evidente en los cinco procesos de formación docente estudiados en este proyecto: comentarios como el presentado al comienzo de esta sección fueron comunes en todas las entrevistas y grupos focales con los actores encargados de la formación docente. En cuatro de los cinco casos estudiados, la visión del proceso se asociaba en alguna medida con la utilización pedagógica de herramientas interactivas y del trabajo virtual. Esta integración incluía la construcción de espacios virtuales para la reflexión pedagógica (e. g., Blogs, Wikis), la construcción de herramientas de aula usando hipertexto y el desarrollo de espacios pedagógicos en Internet para la reflexión sobre diversos temas por parte de los estudiantes. Sin embargo, los actores al nivel de los programas de formación docente reportaron un conflicto permanente entre ese intento de construir una nueva pedagogía de lo virtual y tradiciones más amplias a nivel regional. Esta tensión llegaba a su punto crítico cuando los actores al nivel de decisión regional, en particular en las secretarias de educación, desconocían la relevancia de los programas para la formación docente y para los planes estratégicos del Ministerio.

Los resultados mostraron que la apropiación de una u otra lógica pedagógica no depende exclusivamente de las preferencias en el ambiente institucional regional, sino que también se conecta con factores estructurales de la organización del proceso de formación docente. En particular, es importante que el proceso de formación se organice en varios niveles sucesivos que incluyan la alfabetización tecnológica básica, la construcción de prácticas de aula y la reflexión tecnológica más amplia. Cuando el proceso de formación no está organizado de esta manera, los programas, cuyo objetivo es crear centros de investigación y reflexión pedagógica como el que es objeto de esta investigación, deben concentrarse en la alfabetización tecnológica básica y no pueden evolucionar hacia prácticas más complejas de investigación y desarrollo pedagógico.
El espacio físico de la reforma y los límites de la cognición distribuida

"Fue muy rico el material, conocimos muchos sitios web en los que podíamos encontrar información, portales, foros, logramos aprender no sólo del foro de nosotros sino el de cualquier otra área, pues participábamos con otros grupos, de otros colegios, de otras áreas diferentes a la de uno [...]" (Región 4: entrevista con docente beneficiario).

El tercer punto que se hace evidente en los resultados de este estudio es la dificultad que tienen las teorías de cognición distribuida para impactar en los procesos de reforma a nivel regional. En cuatro de los cinco programas de formación docente se reportó la intención de producir procesos de construcción colectiva de conocimiento usando herramientas virtuales; entre éstos se cuentan el uso de aulas virtuales, tableros virtuales y buscadores para alimentar y enriquecer las prácticas de construcción pedagógica. Sin embargo, sólo uno de estos programas tuvo continuidad y estaba activo al momento de la recolección de datos. En alguna medida, los problemas para darle continuidad a los programas de formación docente pueden ser atribuidos a la distancia existente entre las prácticas de los programas de for- mación y las prioridades de los actores encargados de la decisión a nivel regional. Aunque los programas habían sido elaborados con el Ministerio, siguiendo estrictas directrices pedagógicas, su importancia y calidad no eran percibidas en las regiones; cuando el soporte directo del Ministerio se debilitaba debido a cambios en la agenda y en el personal que llevaba el seguimiento a los proyectos, los centros de formación docente eran percibidos como innecesarios por los encargados de tomar decisiones en las regiones.

\section{Implicaciones: sobre cómo sintonizar la enseñanza con el espíritu de la época}

Piaget (1970) comentó, en referencia a la continua sospecha sobre las máquinas de enseñanza desarrolladas en el paradigma de la tecnología educativa:

Estas máquinas han desempeñado al menos un gran servicio para nosotros, que es el de demostrar mas allá de toda duda posible el carácter mecánico de la función del maestro de escuela tal como es concebida por los métodos tradicionales de enseñanza: si el ideal de ese método es solamente sonsacar la repetición correcta de lo que ha sido

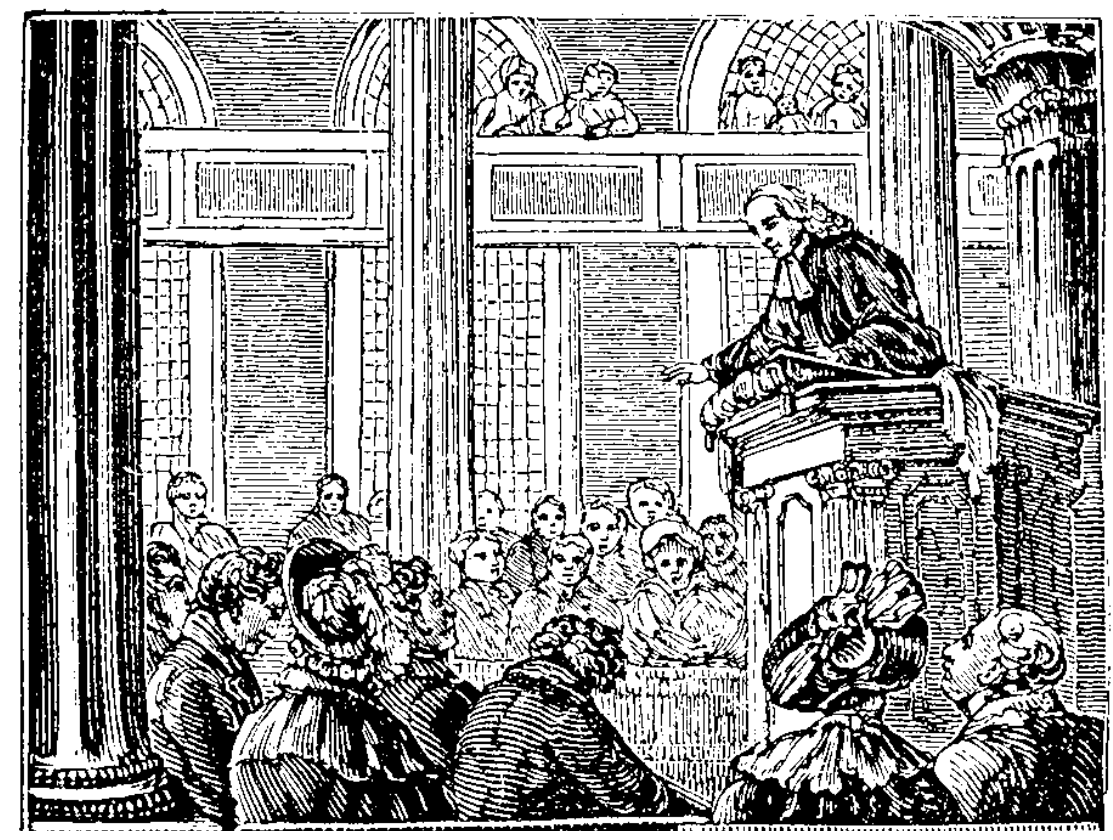


correctamente transmitido, entonces no hay siquiera que aclarar que una máquina puede cumplir correctamente estas condiciones (p. 77).

Está opinión es perfectamente aplicable a los usos de los computadores en la escuela actual.

El punto de la reforma escolar y de la formación docente en e-learning pasa no sólo por la apropiación de la tecnología por parte de docentes y estudiantes, sino por una modificación profunda de las prácticas de enseñanza. Esta modificación requiere, como lo señalan los teóricos de la cognición distribuida y de la perspectiva situada en psicología educativa, construir una educación adecuada a los contextos socioculturales y humanos en los que se produce la actividad y el razonamiento cotidiano de las personas. Estos elementos, las afordancias (affordances), propias de los ambientes culturales de los estu-

\section{BIBLIOGRAFÍA}

Bautista, G. (1999). Capacitación o profesionalización de los maestros en física en ejercicio. TED, 6, pp.14-22.

Berman, P. y McLaughlin, M. (1976), Implementation of educational innovation. The Educational Forum, 40, pp. 345-370.

Coburn, C. (2004). Beyond decoupling: rethinking the relationship between the institutional environment and the classroom. Sociology of Education, 77, pp. 211-244.

Coburn, C. y Talbert, J. (2006). Conceptions of evidence-based practice in school districts: Mapping the terrain. American Journal of Education, 112(4), pp. 469-495.

Cuban, L. (1993), Computers meet classroom: classroom wins. Teachers College Record, 95(2), pp.185-210.

Díaz, O. (1997). Políticas educativas y formación de maestros. Pedagogía y Saberes, 10, pp. 43-51. diantes, deben ser incorporados a las prácticas de aprendizaje para garantizar transferencia de lo aprendido en la escuela a la vida real (Greeno et al, 1996). El estado de cosas en el mundo actual provee herramientas que facilitan las tareas procedurales (e. g., la parte mecánica de despejar una ecuación) y permiten acceso casi inmediato a fuentes de información diversas. Así como la aparición de la escritura modificó la concepción de inteligencia hace siglos (Gardner, 1983), la aparición de los computadores y la Internet deben modificar la nuestra, haciéndola más cercana al paradigma de la cognición distribuida. Esta concepción, que se hace más clara en los espacios de investigación y en los centros de decisión nacional, no se transmite, sin embargo, automáticamente a las prácticas de reforma y formación docente en las regiones.

Los resultados de este estudio muestran la gran distancia existente entre la refor-

DriJvers, P. y Van Herwatrden, O. (2001). Instrumentation of ICT-tools: the case of algebra in a computer algebra environment. International Journal of Computer Algebra in Mathematics Education, 7(4), pp. 255-275.

Espejo, R., Schuhmann, W., Schwaninger, M. y BILELlo, U. (1996). Organizational transformation and learning. Chichester: John Wiley \& Sons.

FIRESTONE, W. (1985). The study of loose coupling: problems, progress, and prospects. Research in Sociology of Education and Socialization, 5, pp. 3-30.

GARDNER, H. (1983). Frames of mind: the theory of multiple intelligences. Nueva York: Basic Books.

Guin, D. Y Trouche, L. (1999). The complex process of converting tools into matheamtical intruments: the case of calculators. International Journal of Computers for Mathematical Learning, 3, pp. 195-227. ma tal como es planeada en los centros de decisión nacional y como es aplicada en las regiones. Se hace claro aquí que en muchos casos estas dificultades surgen de las presiones simultáneas, y en muchos casos contradictorias, a las que tienen que responder los encargados de la reforma a nivel regional y los docentes beneficiarios. Dos lecciones parecen claras: una, que la organización de los procesos de reforma y formación docente en e-learning deben estar organizados en niveles que permitan descargar a los centros de reflexión pedagógica de la alfabetización tecnológica básica; dos, que el músculo político del Ministerio necesita usarse para respaldar y legitimar los programas de formación frente a las autoridades regionales.

Greeno, J., Collins, A. y Resnick, L. (1996). Cognition and learning. En D. Berliner y P. Calfee (eds.), Handbook of educational psychology. Nueva York: Simon \& Schuster.

Halverson, R., Kelley, C. y Kimball, S. (2004). Implementing teacher evaluation systems: how principals make sense of complex artifacts to shape local instructional practice. En W. Hoy y C. Miskel (eds.), Theory and research in educational administration. Greenwich: Information Age Publishing.

HEID, M. (1988). Resequencing skills and concepts in applied calculus using the computer as a tool. Journal for Research in Mathematics Education, 19(1), pp. 3-25.

Herington, J. y Herrington, A. (1998). Authentic assessment and multimedia: How university students respond to a model of authentic assessment. Higher Education Research and Development, 17(3), pp. 305-322. 
Hubbard, L., Stein, M. y Mehan, H. (2006). Reform as learning: when school reform collides with school culture and community politics. Nueva York: Routledge Falmer.

HUtChins, E. (1995). How the cockpit remembers it speed. Cognitive Science, 19(3), pp. 265-288.

JOHNSON-LAIRD, P. (1980). Mental models in cognitive science. Cognitive Science, 4, pp. 71-115.

KINTSCH, W. (1988). The role of knowledge in discourse comprehension: a construction-integration model. Psychological Review, 95, pp.163-182.

KLAHR, D. Y LI, J. (2005). Cognitive research and elementary science instruction: from the laboratory, to the classroom, and back. Journal of Science Education and Technology, 4(2), pp.217-238.

Lovett, M. y Greenhouse, J. (2000). Applying cognitive theory to statistics instruction. The American Statistician, 54, pp. 196-206.

LIGHT, D. et al. (2005). Linking data and learning-The Grow Network StudySummary Report. Nueva York: Educational Development Center's Center for Children and Technology.
MAgUiRE, E. y UCHIDA, C. (2000). Measurement and explanation in the comparative study of american police organizations. En D. Dufeed (ed.), Criminal justice 2000: measurement and analysis of crime and justice (pp. 491-557). Washington: Office of Justice Programs-US Department of Justice.

Matusov, E., Hayes, R. y Pluta, M. (2005). Using discussion web to develop an academic community of learners. Educational Technology \& Society, 8(2), pp.16-39.

TORRES, R. (2000). De agentes de la reforma a sujetos del cambio: la encrucijada docente en América Látina. Perspectivas, 30(2), pp. 281-299.

MEYER, J. y RowAN, B. (1977). Institutional organizations: formal structure as myth and ceremony. American Journal of Sociology, 83, pp. 340-363.

Palincsar, A. y Brown, A. (1984). Reciprocal teaching of comprehensionfostering and comprehension-monitoring activities. Cognition and Instruction, 1(2), pp. 117-175.

Perkins, D. y Salomón, G. (1992). Transfer of learning. En International Encyclopedia of Education (segunda edición). Oxford: Pergamon Press.
Piaget, J. (1970). Science of education and the psychology of the child. Nueva York: Orion Press.

Phillips, D. y SchweIsfuRth, M. (2006), Comparative and international education. An introduction to theory, method and practice. Nueva York: Continuum International Publishing Group.

RowAN, B. y MISKEL, C. (1999). Institutional theory and the study of educational organizations. En J. Murphy y K. S. Louis (eds.), Handbook of research in educational administration (pp. 359-384). San Francisco: Jossey-Bass. pp. 359-384.

ScHuck, S. (2003). The use of electronic question and answer forums in mathematics teacher education. Mathematics Education Research Journal, 5, pp. 19-31.

SIMON, H. y HAYES, J. (1976). The understanding process: problem isomorphs. Cognitive Psychology, 8, pp. 165-190.

Strauss, A. y Corbin, J. (1998). Basics of qualitative research: techniques and procedures for developing grounded theory. Thousand Oaks, California: Sage.

WiLliams, J. (2006). The place of the closed book, invigilated final examination in a knowledge economy. Educational Media Internacional, 43(2), pp. 107-119. 